\title{
DIFICULDADES DE APRENDIZAGEM EM ADULTOS: PSICOANDRAGOGIA COMO ESTRATÉGIA PARA PROPICIAR UMA MELHOR APRENDIZAGEM
}

\author{
Cátia Alessandra Câmara BARBOSA ${ }^{\mathbf{1}}$; Gil Dutra FURTADO²
}

\begin{abstract}
${ }^{1}$ Graduação em Pedagogia/UNP, Especialista em Psicopedagogia/UNP. Pedagoga do NAPe- Núcleo de acessibilidade e apoio psicopedagógico, na Universidade Potiguar-RN, Brasil. Email: catia.gencati@gmail.com

${ }^{2}$ Graduando em Medicina Veterinária UNINASSAU/PB; Engenheiro Agrônomo/UFPB; Doutor em Psicobiologia/Universidade Federal do Rio Grande do Norte (UFRN); Agrônomo-Sócio da Cooperativa de Agronegócio (COOPAGRO), Brasil. E-mail: gdfurtado@hotmail.com
\end{abstract}

Resumo. Este artigo tem como objetivo apresentar uma realidade que requer uma estratégia de intervenção diante das dificuldades que envolvem o ensino e a aprendizagem de adultos e passamos a propor uma profissão que atuaria em exclusividade com este seguimento de pessoas, que seria a "psicoandragogia". Colocando-nos como pesquisadores e observadores, procuramos buscar soluções para reparar as lacunas existentes entre os estudantes da educação superior, lacunas estas que quando observadas podem ser avaliadas e acompanhadas, mediante ações diversas, e resolvidas ao menos em parte, através de um olhar crítico, contribuindo para que o desejo de que a educação especial inclusiva, realmente possa se expressar mediante o direito de igualdade para todos, além das adequações das instituições onde estes desenvolvem suas atividades. Dentro desse contexto inclusivo que abordamos, a "psicoandragogia" é apresentada como uma alternativa para incrementar cada vez mais o bem-estar dos adultos. Utilizando-se de revisões de literatura para melhor entendimento dos efeitos psicológico, sociais e estudantis das dificuldades que muitos adultos são acometidos por variados motivos, direta e indiretamente ligados com o ensino e a aprendizagem, e o levantamentos de dados adquiridos em ambiente superior de estudo, ponderou-se a importância do profissional psicoandragogo, que estaria voltado exclusivamente para atender as demandas de ensino e aprendizado do público adulto, contribuindo para uma melhor adequação da realidade de muitos que estão nesta importante fase de desenvolvimento social e profissional de nossa sociedade. Na conclusão é reforçado a importância deste olhar mais cuidadoso, para essa demanda, a fim de ajudar a fortalecer e tornar essa aprendizagem dos adultos algo mais intrínseco e mais significativo, visto que nunca é tarde para qualquer pessoa aprender; porém que aqueles que têm uma certa dificuldade para isto, sejam ajudados com estratégias mais eficazes e um profissional específico, que é o psicoandragogo.

Palavras chave: Andragogia; Dificuldades de aprendizagem; Inclusão.

\section{DIFFICULTIES FOR LEARNING IN ADULTS: PSYCHOANDRAGOGY AS STRATEGY TO PROVIDE A BEST LEARNING}


Abstract. This article aims to present a reality that requires a strategy of intervention in the face of the difficulties involved in teaching and learning of adults and we propose a profession that would act exclusively with this follow up of people, that would be the "psychoandragogy". By placing ourselves as researchers and observers, we seek to find solutions to address the gaps among higher education students, which, when observed, can be evaluated and monitored through various actions and resolved at least in part through a critical eye, contributing to the desire for inclusive special education to truly express itself through the right of equality for all, in addition to the adequacy of the institutions where they carry out their activities. Within this inclusive context that we address, "psycho-androgyny" is presented as an alternative to increasingly increase the well-being of adults. Using literature reviews to better understand the psychological, social and student effects of the difficulties that many adults face, for reasons directly and indirectly linked to teaching and learning, and the data collection acquired in a higher study environment, it was considered the importance of the professional psycho-drama, which would be exclusively aimed at meeting the demands of teaching and learning of the adult public, contributing to a better adaptation of the reality of many who are in this important phase of social and professional development of our society. In conclusion, the importance of this more careful look at this demand is reinforced in order to help strengthen and make this adult learning something more intrinsic and meaningful, since it is never late for anyone to learn; but that those who have a certain difficulty for this, are helped with more effective strategies and a specific professional, who is the psycho-manager.

Keywords: Andragogy; Learning difficulties; Inclusion.

\section{DIFICULTADES DE APRENDIZAJE EN ADULTOS: PSICOANDRAGOGIA COMO ESTRATEGIA PARA PROPICIAR UN MEJOR APRENDIZAJE}

Resumen. Este artículo tiene como objetivo presentar una realidad que requiere una estrategia de intervención ante las dificultades que involucran la enseñanza y el aprendizaje de adultos y pasamos a proponer una profesión que actuaría en exclusividad con este seguimiento de personas, que sería la "psicoandragogia". En el caso de los investigadores y observadores, buscamos buscar soluciones para reparar las lagunas existentes entre los estudiantes de la educación superior, lagunas éstas que cuando se observan pueden ser evaluadas y acompañadas, mediante acciones diversas, y resueltas al menos en parte, a través de una mirada crítica, contribuyendo para que el deseo de que la educación especial inclusiva, realmente pueda expresarse mediante el derecho de igualdad para todos, además de las adecuaciones de las instituciones donde éstos desarrollan sus actividades. Dentro de ese contexto inclusivo que abordamos, la "psicoandragogia" es presentada como una alternativa para incrementar cada vez más el bienestar de los adultos. El uso de revisiones de literatura para entender mejor los efectos psicológicos, sociales y estudiantiles de las dificultades que muchos adultos son acometidos por variados motivos, directa e indirectamente ligados con la enseñanza y el aprendizaje, y el levantamiento de datos adquiridos en un ambiente superior de estudio, se ponderó la importancia del 
profesional psicoandragogo, que estaría orientado exclusivamente a atender las demandas de enseñanza y aprendizaje del público adulto, contribuyendo a una mejor adecuación de la realidad de muchos que están en esta importante fase de desarrollo social y profesional de nuestra sociedad. En la conclusión se refuerza la importancia de esta mirada más cuidadosa, para esa demanda, a fin de ayudar a fortalecer y hacer que ese aprendizaje de los adultos algo más intrínseco y más significativo, ya que nunca es tarde para cualquier persona aprender; pero que aquellos que tienen una cierta dificultad para esto, sean ayudados con estrategias más eficaces y un profesional específico, que es el psicoandragogo.

Palabras clave: Andragogía; Dificultades de aprendizaje; Inclusión.

\section{INTRODUÇÃO}

Atualmente as discussões sobre as dificuldades de aprendizagem no meio acadêmico sinalizam que os professores que atuam com essa demanda devem se debruçar sobre estudos cada vez mais minuciosos de competências e habilidades necessárias a serem introduzidas ao saber docente dentro desse contexto (NOFFS; RODRIGUES, 2011).

Por ver crescer a cada dia uma grande parte dessa população discente que está postergando essa situação e por vivermos com o paradigma de não existir muitas leituras sobre como lidar com essas situações nos mostra o surgimento do momento em que se sabe que estes não podem viver sempre com o insucesso acadêmico, tornando-se salutar encontrar o equilíbrio necessário (NOFFS; RODRIGUES, 2011).

A convicção de que cada pessoa que convive com um déficit se sente confortável, sofrendo as consequências disso, nos mostra que na maioria das vezes esses sujeitos nem sabem que têm esses déficits, até porque sempre foram rotulados desde a infância como lentos, desatentos, dentre outros.

A demanda experimental proporcionou observar adultos em uma faculdade particular (NAAPe- Núcleo de apoio e acompanhamento psicopedagógico), onde foram realizados só no primeiro semestre 76 atendimentos e avaliados 10 patologias.

Esta pesquisa traz a luz educacional um contexto geral e globalizado, onde quando se buscam rumos para estudar o jovem e o adulto com déficits, em geral, se evidencia que, nas academias e nas literaturas psicopedagógicas, ainda pouco se fala dessa parte da população, focando-se muito no objetivo de resolver os problemas ligados aos jovens e crianças, ficando, por outro lado, os adultos desamparados (KNOWLES; HOLTON III; SWANSON, 1980).

Foi observado também que no estágio clínico, profissionais psicopedagogos que atendem adultos não sabem muito bem como agir para com esses sujeitos, pois as atividades e 
metodologias para se trabalhar dificuldades de aprendizagem são geralmente voltadas às crianças (KNOWLES; HOLTON III; SWANSON, 1980).

Faz-se necessário a construção de metodologias e práticas educacionais voltadas ao ensino da andragogia, visando melhorar a qualidade de vida desses sujeitos nas escolas, faculdades, em casa, com seus amigos, relacionamentos e no trabalho. Segundo Noffs e Rodrigues (2011), o modelo aplicado à educação de adultos, deve desenvolver-se no foco do "como este aprende", seguindo uma lógica que perpassa pela sensibilização, experimentação, pesquisa, discussão, conclusão e finalmente compartilhamento (NOFFS; RODRIGUES, 2011).

Assim como na psicopedagogia se faz com as crianças, aplicando diagnósticos e intervenções, usando todas as ferramentas psicopedagógicas, seguindo etapas de: Entrevista Familiar Exploratória Situacional (E.F.E.S), entrevista de Anamnese, sessões lúdicas centradas na aprendizagem, complementação com provas e testes (quando necessário), síntese diagnóstica - Prognóstico, entrevista de Devolução e Encaminhamento (MORAES, 2010), a psicoandragogia, surge como uma atividade profissional que diz respeito as estratégias educacionais estabelecidas para adultos, sugerindo a partir dos conhecimentos pedagógicos, psicopedagógicos, a interdisciplinaridade e a vivência no ensino superior, criar ferramentas metodologias e práticas educacionais para levar o adulto a saber caminhar de forma madura ao reencontrar o seu novo percurso, usando outras formas de averiguar e fazer o levantamento de seus próprios dados, superando as dificuldades (FURTADO, 2016; NOFFS; RODRIGUES, 2011).

Considerando os adultos, parte da população menos favorecida por um acompanhamento mais específico para os "déficits de ensino e aprendizado", uma realidade que vem se mostrando cada vez mais presente na sociedade, é que se desenvolve a temática desta pesquisa e estudo na visão psicoandragógica para trabalhar com estes casos.

A integração com as outras áreas afins é de suma importância, visto que tudo sobre déficit de aprendizado engloba uma série de informações de diferentes focos científicos e precisa ser tratado com o envolvimento de vários profissionais (FURTADO; SANTOS, 2015).

O objetivo deste trabalho procura mostrar a importância do profissional psicoandragógico direcionando para os défices educacionais específicos dos adultos. 


\section{MATERIAL E MÉTODOS}

Foi realizada uma revisão bibliográfica direcionada, com objetivo de direcionar as ações de observação e interpretação dos resultados, usando metodologia que envolve conhecimento na vida da comunidade estudada. Daí por que se pode definir observação participante como a técnica pela qual se chega ao conhecimento da vida de um grupo a partir do interior dele mesmo (GIL, 2002).

A pesquisa procedeu-se mediante observações participante com acompanhamento de alunos universitários e avaliações feitas por psicopedagogos. Para confirmar os resultados das observações, realizou-se revisões bibliográficas em periódicos, livros e artigos científicos publicados interdisciplinarmente (GIL, 2002).

\section{RESULTADOS E DISCUSSÃO}

Em tempos modernos a transformação do mercado de trabalho tem demandado profissionais que possuam curso superior. No nosso país é observado uma crescente onda de faculdades que tem o objetivo de atender está forte demanda da sociedade e com isso mais pessoas tem tido a oportunidade de se qualificarem em diversas áreas de atuação.

Com esta abertura de oportunidades de estudos, as pessoas têm ficado mais expostas quanto a suas dificuldades de ensino e aprendizagem, gerando um anseio muito grande perante a sociedade, família e a si próprio, por descobrir limites, complicações e desafios que jamais teria sido revelado se não fosse esta situação de qualificação por meio de estudos em ambientes de ensino superior.

Longe de estes serem limitantes para os adultos, este artigo procura apresentar o resultado de observações e pesquisa desenvolvidos dentro desta realidade educacional, e durante o período de coleta de dados, que foi de seis messes, chegou-se aos seguintes resultados:

O universo amostral foram alunos oriundos de 8 cursos distintos, que totalizaram 76 indivíduos, sendo estes cursos:

1) $28,95 \%$ de medicina;

2) $23,68 \%$ de fonoaudiologia;

3) $19,74 \%$ de educação física;

4) $9,21 \%$ de odontologia;

5) 7,89\% de veterinária; 
6) $5,26 \%$ de nutrição;

7) $3,95 \%$ de tec. de saúde bucal;

8) $1,32 \%$ de fisioterapia.

E um total de identificação e acompanhamento de 10 patologias, que foram:

1) $27,14 \%$ déficit de aprendizado;

2) $18,57 \%$ síndrome de Down;

3) $11,43 \%$ dislexia;

4) $10 \%$ déficit de rotina;

5) $10 \%$ saúde mental;

6) $5,71 \% \mathrm{TDA} / \mathrm{H}$ (Transtorno do Déficit de Atenção com Hiperatividade);

7) $5,71 \%$ de déficit de aprendizado/TODA (Transtorno do Déficit de Atenção);

8) $4,29 \%$ de saúde mental / concentração;

9) $4,29 \%$ déficit de concentração;

10) $2,86 \%$ de paralisia cerebral.

Os resultados observados acima indicam que as dificuldades de aprendizagem estão bem presentes no cotidiano dos adultos, indicando a importância que se tem em desenvolver metodologias específicas para atender e estes, que fazem parte da sociedade produtiva (no tocante a força de trabalho) e que são responsáveis em sua realidade social familiar e de trabalho, além de serem parte dos futuros profissionais que formarão e direcionaram o desenvolvimento técnico profissional qualificado com títulos de cursos "superior" e por isso terão forte impacto na sociedade brasileira. Também não se pode esquecer que em qualquer ambiente onde tenha qualquer tipo de "ensino e aprendizado" o psicoandragogo será de suma importância para os adultos, independentemente de qualquer fator biológico, social, financeiro, dentre outros (FURTADO, 2016).

Com o fomento para que ocorra uma ampliação das micros e pequenas empresas, também se observa que a temática de ensino e aprendizado tem se ampliado, pois para todas as atividades que se inicia, ocorre a ação direta e/ou indireta da arte de ensinar e de aprender, e a psicoandragogia teria sua importância bem expressa, contribuindo para o aprimoramento das empresas, e contribuindo para uma maior garantia dos avanços sociais esperados (RODRIGUES, 2000). 
A psicoandragogia é a parte da psicologia da aprendizagem que vem a estudar a parte do processo de aprendizagem e as estruturas mentais do estudante adulto (ALMEIDA; SILVA, 2005) na qual a sua metodologia está voltada a aprendizagem pela autonomia, experiência e vivência que o sujeito já as possui e assim poderão transformá-las e assimilá-las ao conteúdo. É uma adaptação da vivência do adulto aos conhecimentos de forma mais criativa e prática. Visando melhorar a atuação dos psicopedagogos nos trabalhos com adultos que possuem realidades diferentes das crianças a "psicoandragogia" vem com uma proposta mais acurada, buscando conhecimentos em outros campos, porém criando sua própria área de atuação, baseando-se em áreas multidisciplinares como a neurologia, psicologia, psicanálise, linguística, fonoaudiologia, pedagogia dentre outras, para obter melhores resultados a partir das suas contribuições (FURTADO; SANTOS, 2015).

A psicoandragogia poderá atuar, dentre outras áreas, na falta da percepção que o diagnóstico de transtornos de déficits de atenção quando criança se alastra e passa despercebida ou mascarada pelo período da adolescência até a fase adulta, englobando diferentes problemas para a vida do adulto nos âmbitos pessoal, institucional e social.

O estudo da psicoandragogia está estruturado em torno do processo de aprendizagem, em seus padrões de evolução normais e patológicos, e da influência do meio em seu desenvolvimento; como esse processo é contínuo, o sujeito adulto, necessita encontrar-se dentro deste, para que venha a dar continuidade de forma mais fluente.

O psicoandragogo, num primeiro momento, deverá estar voltado para o desenvolvimento de metodologias que melhor o habilite a trabalhar essas dificuldades, sendo esta, melhor adaptada à realidade desses sujeitos, buscando melhorar a relação com sua própria aprendizagem visto que o adulto, neste caso, busca um sentido para saber as razões do porquê da sua dificuldade de aprendizagem, e buscar aplicações práticas para solucioná-las (FURTADO et al., 2017; FURTADO; CÂMARA; COSTA, 2017)

De meados dos anos 60 ao início dos anos 70, começavam a ser difundidas as dificuldades de aprendizagem, isso nos faz pensar que as crianças que tinham dificuldades de aprendizagem, nessas décadas, são hoje adultos convivendo com as consequências de tais dificuldades nos relacionamentos afetivos, familiares, sociais e no trabalho.

Provavelmente são pessoas que não conseguem se encontrar numa profissão (como se tem confirmado com alguns indivíduos acompanhados), tem dificuldades nos relacionamentos, fizeram diversas atividades ao mesmo tempo sem conseguir finalizá-las, fazem vários cursos (na maioria das vezes sem um ter nada a ver com o outro) e não conseguem terminar nenhum (FURTADO; CÂMARA; COSTA, 2017). 
Não é difícil encontrarmos pessoas nas clínicas de psicólogos, psicanalistas, neurologistas, psiquiatras, dentre outros, relatando casos de insucesso acadêmico ou mesmo em casa, nos relacionamentos, nas amizades, de forma frustrada, com baixa autoestima, depressão, cansaço físico e mental, por viverem uma eterna batalha solitária e o pior, vista de forma distorcida por muitas pessoas. Realmente, no caso do trabalho com jovens e adultos é fundamental que haja uma equipe multidisciplinar de avaliação e acompanhamento, pois são questões já arraigadas no sujeito em questão.

Profissionais que trabalham com adultos há muitos anos em cursos técnicos e em graduações universitárias percebem essas dificuldades, levando-os a pesquisar como trabalhar a aprendizagem de uma forma mais voltada às suas realidades, pois o que é aplicado para as crianças não será eficiente para eles (FURTADO; CÂMARA; COSTA, 2017). Como nos casos os cursos são profissionalizantes, envolvem uma série de situações estressantes como responsabilidades e ansiedades que engloba tanto o emocional quanto seu futuro profissional.

Considerando tais fatores, a atuação do profissional "psicoandragogo" visa atender as expectativas de amenizar as dificuldades que estes sujeitos apresentam, tirando-os de uma situação complicada na qual se encontram em suas vidas e proporcionando uma melhor percepção do momento presente, sem muitas interferências externas, favorecendo seu processo de aprendizagem e sociabilização com a sociedade em geral.

Os desafios, neste caso, são muitos pois estes sujeitos chegam para os profissionais com suas experiências de vida bem constituídas e diante de tais fatos podemos concluir que o atendimento e acompanhamento feitos por um psicoandragogo é de grande importância, envolvendo grande responsabilidade, tendo em vista que os adultos com déficit têm consciência do que querem, porém não possuem muita clareza do problema que têm. Necessitam compreender o processo que estão envolvidos no atendimento porque esperam que realmente os atendimentos lhes tragam melhorias e que esse novo vínculo com a aprendizagem possa ser uma experiência realmente melhor para sua vida (FURTADO; CÂMARA; COSTA, 2017).

\section{CONCLUSÕES}

Diante do exposto e sabendo a importância de se trabalhar as dificuldades de aprendizagem em adultos, que é uma demanda crescente, necessitam-se de um olhar mais filtrado e um apoio através de um atendimento específico nessa faixa etária para superar todos os agravos no percurso de vida. Percebe-se a importância de pesquisar minuciosamente estratégias que 
venham contribuir para uma melhor qualidade no que se diz respeito ao sujeito adulto aprendiz.

A pouca literatura sobre tal assunto (não nos referindo apenas à andragogia no seu aspecto geral que é o de ensinar adultos) e nas especificidades de cada caso, é que nos faz levantar a questão: "Como trabalhar adultos com dificuldades de aprendizagem?".

Podemos dizer que estão em toda parte, a começar pelas universidades, onde estes se deparam com as dificuldades na sua forma mais difícil que envolve questões muito mais significantes para eles, pois se trata da importante atividade de acompanhar as disciplinas, as turmas de estudo, e principalmente a incerteza da sua futura capacidade profissional, visto que eles vivem o insucesso universitário.

Desta forma, reforçamos a importância deste olhar mais cuidadoso, para essa demanda, a fim de ajudar a fortalecer e tornar essa aprendizagem algo mais intrínseco e mais significativo, visto que nunca é tarde para qualquer pessoa aprender; porém que aqueles que têm uma certa dificuldade para isto, sejam ajudados com estratégias mais eficazes e um profissional específico, que é o psicoandragogo.

\section{REFERÊNCIAS}

ALMEIDA, F.; SILVA, M.B. Psicopedagogia para adultos - psicoandragogia: uma proposta de atendimento psicopedagógico para adolescentes e adultos jovens. Revista Universitária de Biologia e Saúde (RUBS), Curitiba, v. 1, n. 4, p.46-48, out/dez. 2005. Supl. 1.

FURTADO, G.D. A importância da atuação do psicopedagogo nos programas de pós-graduações. In: Fernando Abadh Cananéa. (Org.). Educação (Re) discussões (Im) pertinentes. $1^{\mathrm{a}}$ ed. João Pessoa: Imprell, 2016, v. 1, p. 121-134.

FURTADO, G.D.; BATALLA, J.F.; COSTA, D.A.; CÂMARA, C.A.B. Relato descritivo de uma intervenção psicopedagógica clínica. Educação Ambiental em Ação, v. 59, ano 15, p. 1-10, mar/mai. 2017.

FURTADO, G. D.; CÂMARA C.A.B.; COSTA, D.A. Avaliação psicopedagógica discente em uma pós-graduação na paraíba. Revista Etos, v. 1, n. 1, p. 116-127, jan/jun. 2017. 
FURTADO, G.D.; SANTOS K.C.S. Epistemologia do educando cognoscente sob estresse psicológico prolongado: um estudo de caso. Revista Intersaberes, v. 10, n. 20, mai./ago. 2015.

GIL, A.C. Como elaborar projetos de pesquisa. 4. ed. São Paulo: Atlas, 2002.

KNOWLES, M.S.; HOLTON III, E.F.; SWANSON, R.A. The modern practice of adult education: from pedagogy to andragogy. New York: Association Press; 1980.

MORAES, D.N.M. Diagnóstico e avaliação psicopedagógica. Revista de Educação do IDEAU (REI), Rio Grande do Sul, v. 5, n. 10, jan/jun. 2010. Disponível em: https://www.ideau.com.br/getulio/restrito/upload/revistasartigos/203_1.pdf. Acesso em: 26 nov. 2018.

NOFFS, N.A.; RODRIGUES, C.M.R. Andragogia na Psicopedagogia: a atuação com adultos. Revista Psicopedagogia, São Paulo, v. 28, n. 87, p. 283-292, 2011. Disponível em: http://pepsic.bvsalud.org/scielo.php?script=sci_arttext\&pid=S010384862011000300009\&lng=pt\&nrm=iso. Acesso em: 23 nov. 2018.

RODRIGUES, M.E. O conhecimento nas micro e pequenas empresas: um estudo sobre sua absorção e utilização nas micro e pequenas empresas fluminenses. 2000. 175 f. Dissertação (Mestrado em Administração) - Universidade Federal do Rio de Janeiro, Rio de Janeiro, 2000 . 